\title{
Nutritional Efficiency in Clones of Conilon Coffee for Phosphorus
}

\author{
Lima Deleon Martins ${ }^{1}$, Marcelo Antonio Tomaz ${ }^{1}$, José Francisco Teixeira do Amaral ${ }^{1}$, Scheilla Marina Bragança ${ }^{2}$, \\ Wagner Nunes Rodrigues ${ }^{1} \&$ Edvaldo Fialho dos Reis ${ }^{1}$ \\ ${ }^{1}$ Universidade Federal do Espírito Santo, Centro de Ciências Agrárias, Programa de Pós-Graduação em Produção \\ Vegetal, Alegre, Espírito Santo, Brazil \\ ${ }^{2}$ Instituto Capixaba de Pesquisa, Assistência Técnica e Extensão Rural, Linhares, Espírito Santo, Brazil \\ Correspondence: Lima Deleon Martins, Universidade Federal do Espírito Santo, Centro de Ciências Agrárias, \\ Programa de Pós-Graduação em Produção Vegetal, Alegre, Espírito Santo, Brazil. Tel: 55-283-552-8998. E-mail: \\ deleon_lima@hotmail.com
}

Received: September 25, 2012 Accepted: October 22, 2012 Online Published: December 13, 2012

doi:10.5539/jas.v5n1p130

URL: http://dx.doi.org/10.5539/jas.v5n1p130

\begin{abstract}
The objective of this study was to evaluate the nutritional efficiency of phosphorus in clones of conilon coffee, in greenhouse condition. A factorial design $13 \times 4$ was used, with three replications, and the factors being: 13 clones that compose the clonal cultivar "Vitória Incaper 8142" and four levels of phosphorus fertilization ( $0 \%$, $50 \%, 100 \%$ and $150 \%$ of the recommended $\mathrm{P}_{2} \mathrm{O}_{5}$ for the culture) in a completely randomized design (CRD). The cultivation consisted of a period of 150 days, then in each experimental unit, the values of dry matter and phosphorus content of the vegetative parts were obtained. The indices of nutritional efficiency (absorption, translocation and utilization) were calculated. The clones of conilon coffee showed different behavior for all variables at each level of fertilization with $\mathrm{P}_{2} \mathrm{O}_{5}$. The mass of dry weight, phosphorus content, and efficiency of phosphorus utilization of the clones increase linearly with the increase of $\mathrm{P}_{2} \mathrm{O}_{5}$. The efficiency of phosphorus absorption, depending on the levels of $\mathrm{P}_{2} \mathrm{O}_{5}$ applied to the clones of conilon coffee, has set to a quadratic model. The translocation efficiency of phosphorus, depending on the levels of $\mathrm{P}_{2} \mathrm{O}_{5}$ applied, is different for clones of conilon coffee, with a linear characteristic and also quadratic.
\end{abstract}

Keywords: coffea canephora, mineral nutrition, utilization efficiency

\section{Introduction}

Broadly, the concept of nutritional efficiency is used to characterize the ability of a given genotype to absorb the nutrient and then use it for the production of biomass or vegetal material of economic importance (Furtini Neto, 1994). In tropical soils, phosphorus (P) is the nutrient that limits the biomass production the most, due to the source material and the strong interaction of this nutrient with the soil particles (Novais \& Smyth, 1999; Novais et al., 2007; Oladiran et al., 2012). This fact makes the fertilization with P a constant need, thereby raising the production costs (Reis et al., 2011).

Thus, in experiments about the nutritional efficiency, studies regarding the $\mathrm{P}$ should be prioritized in order to increase the efficiency of this nutrient in the crops (Machado et al., 2001), coupled with the economic premise of reducing the use of phosphate fertilizers (Amaral et al., 2012).

It has been observed for most cultures, that differential behavior between the inter and intra-specific for the absortion, translocation, and utilization of $\mathrm{P}$, which mostly happens under genetic influence (Martinez et al., 1993; Fageria, 1998; Machado et al., 2001; Moura et al., 2001; Castro, 2009; Amaral et al., 2012).

The production of conilon coffee in Brazil has been increasing, and the State of Espírito Santo is the largest producer in the country. This is mainly the result of the utilization of new cultivars, in special the clonal cultivar "Vitória Incaper 8142" (Ferrão et al., 2011).

This cultivar, formed by a selected group of 13 clones, excelled the others in relation to a series of features, highlighting the average yield of 70.3 bags of 60 kilograms of green coffee per hectare, exceeding the average of other cultivars recommended for the Espírito Santo State in 21.05\% (Fonseca et al., 2004). However, it is known that cultivars of conilon coffee present high genetic variability (Ferrão et al., 2008) which raises the hypothesis that different clones may have different nutritional requirements. 
Thus, the evaluation of the nutritional efficiency, for phosphorus, in the clonal cultivar "Vitória Incaper 8142", becomes essential to exploit the high yield potential and to reduce the cost of production. This study will, with the advancement of research, enable the recommendation of fertilization differentiated by clone, since they are separated and planted in rows in proportional amounts.

The objective of this study was to evaluate the influence of phosphorus levels in the nutritional efficiency of clones of conilon coffee for phosphorus, in greenhouse condition.

\section{Material and Methods}

\subsection{Description of the Study Area and Plant Material}

The experiment was conducted in greenhouse, located in latitude $20^{\circ} 45^{\prime} \mathrm{S}$, longitude $41^{\circ} 33^{\prime} \mathrm{W}$ and an average elevation of 277.41 meters above sea level. The typical climate of the region is classified as CWa by the Köppen system, presenting hot and humid summers and dry winters. Along the experimentation period, the means for maximum and minimum temperatures were $39.27^{\circ} \mathrm{C}$ and $25.60^{\circ} \mathrm{C}$, respectively, from the months of October 2010 to March 2011.

The soil used was collected at 10 to $40 \mathrm{~cm}$ of depth, discarding the first $10 \mathrm{~cm}$ of the soil profile in order to reduce the effect of organic matter present in the surface layer. A sample of the soil was taken to laboratory for chemical and physical analyzes (Table 1), and the soil was characterized as being Oxisol with clayey texture (Embrapa, 2006).

Table 1. Physical and chemical attributes properties of the soil used as substratum

\begin{tabular}{lc}
\hline Attributes & Values \\
\hline Sand $\left(\mathrm{g} \mathrm{kg}^{-1}\right)^{1}$ & 552.40 \\
Silt $\left(\mathrm{g} \mathrm{kg}^{-1}\right)^{1}$ & 43.60 \\
Clay $\left(\mathrm{g} \mathrm{kg}^{-1}\right)^{1}$ & 403.40 \\
Soil density $\left(\mathrm{kg} \mathrm{dm}^{-3}\right)^{2}$ & 1.20 \\
$\mathrm{pH}^{3}$ & 5.40 \\
$\mathrm{P}\left(\mathrm{mg} \mathrm{dm}^{-3}\right)^{4}$ & 2.00 \\
$\mathrm{~K}\left(\mathrm{mg} \mathrm{dm}^{-3}\right)^{5}$ & 93.00 \\
$\mathrm{Ca}\left(\mathrm{cmol}_{\mathrm{c}} \mathrm{dm}^{-3}\right)^{6}$ & 1.70 \\
$\mathrm{Mg}\left(\mathrm{cmol}_{\mathrm{c}} \mathrm{dm}^{-3}\right)^{6}$ & 1.10 \\
$\mathrm{Al}\left(\mathrm{cmol}_{\mathrm{c}} \mathrm{dm}^{-3}\right)^{7}$ & 0.00 \\
$\mathrm{H}+\mathrm{Al}\left(\mathrm{cmol}_{\mathrm{c}} \mathrm{dm}^{-3}\right)^{8}$ & 2.10 \\
Sum of Bases $\left(\mathrm{cmol}_{\mathrm{c}} \mathrm{dm}^{-3}\right)$ & 3.37 \\
Potencial cation Exchange capacity $\left(\mathrm{cmol}_{\mathrm{c}} \mathrm{dm}^{-3}\right)$ & 5.45 \\
Actual cation Exchange capacity $\left(\mathrm{cmol}_{\mathrm{c}} \mathrm{dm}^{-3}\right)$ & 3.37 \\
Base saturation $(\%)$ & 61.80 \\
\hline
\end{tabular}

Note: ${ }^{1}$ Pipette method (slow agitation); ${ }^{2}$ Beaker method; ${ }^{3} \mathrm{pH}$ in water (ratio 1:2.5); ${ }^{4}$ Extracted by Mehlich 1 and determined by colorimetry; ${ }^{5}$ Extracted by Mehlich 1 and determined by flame photometry; ${ }^{6}$ Extracted with potassium chloride $1 \mathrm{~mol} \mathrm{~L}^{-1}$ and determined by titration; ${ }^{7}$ Extracted with potassium chloride $1 \mathrm{~mol} \mathrm{~L}^{-1}$ and determined with atomic absorption spectrophotometer; ${ }^{8}$ Extracted with calcium acetate $0.5 \mathrm{~mol} \mathrm{~L}^{-1}, \mathrm{pH} 7.0$ and determined by titration (Embrapa, 1997).

After characterization, the entire volume of soil was dried under shade and homogenized in a $2.0 \mathrm{~mm}$ mesh sieve. Subsequently, samples of $10 \mathrm{dm}^{3}$ were separated, by weighing on a precision balance, and placed in sealed plastic pots $(14 \mathrm{~L})$.

\subsection{Expremental Design and Conduct of the Study}

The experiment was conducted in a $13 \times 4$ factorial scheme, with three replications, and the factors being: 13 clones that compose the clonal cultivar "Vitória Incaper 8142" (CV-01, CV-02, CV-03, CV-04, CV-05, CV-06, 
CV-07, CV-08, CV-09, CV-10, CV-11, CV-12 and CV-13) and four levels of phosphorus fertilization (0\%, 50\%, $100 \%$ and $150 \%$ of the recommended level of $\left.\mathrm{P}_{2} \mathrm{O}_{5}\right)$ in a completely randomized design. The recommendation level of $\mathrm{P}_{2} \mathrm{O}_{5}$ was determinate according with the proposed for Lani et al. (2007). The experimental plot consisted of a seedling of each genotype per pot.

The $\mathrm{P}_{2} \mathrm{O}_{5}$ levels corresponding to each experimental plot were applied in the form of p.a. salts (pure for analysis) $\left(\mathrm{KH}_{2} \mathrm{PO}_{4}\right)$, diluted in distilled water and homogenized completely the volume of soil in the pot. The applied levels; referring to $0 \%, 50 \%, 100 \%$ and $150 \%$ of the $\mathrm{P}_{2} \mathrm{O}_{5}$ recommended for culture, according to Lani et al. (2007); consisted of $0.00,3.15,6.30$ and $9.45 \mathrm{~g} \mathrm{pot}^{-1}$ of $\mathrm{P}_{2} \mathrm{O}_{5}$. After application of the levels of $\mathrm{P}_{2} \mathrm{O}_{5}$, the plantation of conilon coffee seedlings, with three pairs of leaves, was performed. The seedlings were provided by the Instituto Capixaba de Pesquisa, Assistência Técnica e Extensão Rural-INCAPER.

The amount of potassium provided to all parcels in a single dose at planting, was $5.20 \mathrm{~g} \mathrm{pot}^{-1}$ of $\mathrm{K}_{2} \mathrm{O}$. The nitrogen fertilization, with $\mathrm{NH}_{2} \mathrm{CONH}_{2}$ P.A., was performed according to Lani et al. (2007), using nitrogen diluted in distilled water and applied on the surface, following a circular in shape, at $10 \mathrm{~cm}$ away from the steam. The fertilization (17.3 g N per pot) was divided into five applications, the first at the day of planting and the other periodically at $30,60,90$ and 120 days after planting.

The irrigation was performed keeping the soil moisture at $60 \%$ of the total pore volume, calculated using the particle density and soil density, determined by the beaker method according to Embrapa (1997).

\subsection{Evaluation of the Study and Calculate Indices}

After 150 days of culture, the plants were cut, separating the stem and branches, leaves and roots. These vegetal parts were removed, washed, weighed and dried in shade. All these parts were separately packed in paper bags and dried in a stove, with forced air circulation, at a temperature of $65^{\circ} \mathrm{C}$, until constant weight, to determine the variables of dry matter.

To determine the mass of dry matter of aerial part (DMAP) and the dry matter of roots (DMR), the material was weighed on precise analytical balance, resulting in the values of grams per plant. The DMAP representing the sum of the weight of the dry mass of leaves, stems and branches. The total dry matter (TDM) was obtained by the sum of the DMAP and the DMR.

Utilizing the dry matter and the content of nutrients in the vegetal tissues, obtained using the methods described by Embrapa (1997), the following indices were calculated: (a) absorption efficiency (AE) $=($ total content of nutrient in the plant)/(root dry matter), according to Swiader et al. (1994); (b) translocation efficiency $(\mathrm{TE})=[($ content of the nutrient in the aerial part $) /($ total content of nutrient in the plant) $] \times 100$, according to Li et al. (1991); and (c) utilization efficiency (UE) $=(\text { total dry matter produced })^{2} /($ total content of nutrient in the plant), according to Siddiqi and Glass (1981).

\subsection{Statistical Analysis}

The data were subjected to analysis of variance $(\mathrm{p} \leq 0.05)$, using the statistical software SISVAR (Ferreira, 2008) and, when significant, the Scott-Knott test $(\mathrm{p} \leq 0.05)$ was used for qualitative factors and regression analysis to quantitative factors. The regression models were chosen based on the significance of the regression coefficients, using the Student $t$ test at $5 \%$ probability and the coefficient of determination $\left(\mathrm{R}^{2}\right)$.

\section{Results and Discussion}

\subsection{Phosphorus Levels Influence Production of Dry Matter and Content of the Phosphorusof Clones of Conilon Coffee}

The factorial analysis of variance revealed the significance of the interaction between genotypes and phosphorus levels. In the study of the variables of dry matter (DMR, DMAP and TDM) (Table 2) and P content (PR, PAP and TP) (Table 3), differential behaviors were observed between the genotypes. Distinct groups of homogeneous means were observed, with different compositions, within each level of phosphorus fertilization studied.

Overall, it can be seen that clone CV-09, at levels of 0 to $100 \%$ of the recommended $\mathrm{P}_{2} \mathrm{O}_{5}$, and clones CV-05, CV-06 and CV-07, in the levels of 100 and $150 \%$, maintained themselves up in the groups of higher average for all variables of dry matter. Contrary, clones CV-10, CV-11 and CV-12 and the clones CV-02 and CV-04, respectively, at levels of 0 to $50 \%$ and 100 to $150 \%$ of the recommended $\mathrm{P}_{2} \mathrm{O}_{5}$, were allocated in the groups of inferior means for those same variables (Table 2).

It is stated by Fageria (1998) that is commonly observed differenced accumulation of dry matter under the same conditions of soil fertility, for cultivars of the same species. This differential behavior is justifiable due to the nutritional requirement being variable between genotypes of the same species, depending on the genetic and 
phenotypic variability (Fageria, 1998). According to Fonseca et al. (2004) and Ferrão et al. (2008), conilon coffee clones have a wide phenotypic and genetic variability, which allows different behavior of the genetic material, indicating the need for attention to adaptability and stability of genotypes for different soil conditions.

Table 2. Means of dry matter of the clones of conilon coffee that compose the clonal cultivar "Vitória Incaper 8142 " for each level of phosphorus fertilization $\left(0,50,100\right.$ and $150 \%$ of the recommended level of $\mathrm{P}_{2} \mathrm{O}_{5}$ for the culture)

\begin{tabular}{|c|c|c|c|c|c|c|}
\hline \multirow{2}{*}{ Clone } & $\mathrm{DMR}^{1}$ & $\mathrm{DMAP}^{2}$ & $\mathrm{TDM}^{3}$ & $\mathrm{DMR}^{1}$ & DMAP $^{2}$ & $\mathrm{TDM}^{3}$ \\
\hline & \multicolumn{3}{|c|}{$0 \%$ of $\mathrm{P}_{2} \mathrm{O}_{5}$} & \multicolumn{3}{|c|}{$50 \%$ of $\mathrm{P}_{2} \mathrm{O}_{5}$} \\
\hline CV-01 & $7.37 \mathrm{c}$ & $34.07 \mathrm{~b}$ & $41.41 \mathrm{~b}$ & $15.17 \mathrm{a}$ & $43.24 \mathrm{~b}$ & $59.01 \mathrm{~b}$ \\
\hline $\mathrm{CV}-02$ & $7.33 \mathrm{c}$ & $22.94 \mathrm{f}$ & $31.42 \mathrm{e}$ & $13.47 \mathrm{~b}$ & $37.18 \mathrm{~d}$ & $50.60 \mathrm{~d}$ \\
\hline $\mathrm{CV}-03$ & $6.10 \mathrm{~d}$ & $28.59 \mathrm{~d}$ & $34.71 \mathrm{~d}$ & $11.63 \mathrm{c}$ & $36.06 \mathrm{~d}$ & $47.78 \mathrm{e}$ \\
\hline CV-04 & $5.57 \mathrm{~d}$ & $26.83 \mathrm{e}$ & $32.39 \mathrm{e}$ & $9.08 \mathrm{e}$ & $32.69 \mathrm{e}$ & $41.89 \mathrm{~g}$ \\
\hline CV-05 & $9.67 \mathrm{~b}$ & $27.73 \mathrm{~d}$ & $37.38 \mathrm{c}$ & $10.77 \mathrm{~d}$ & $34.27 \mathrm{e}$ & $44.93 \mathrm{f}$ \\
\hline CV-06 & $5.73 \mathrm{~d}$ & $26.34 \mathrm{e}$ & $32.06 \mathrm{e}$ & $15.10 \mathrm{a}$ & $43.89 \mathrm{~b}$ & $58.90 \mathrm{~b}$ \\
\hline CV-07 & $9.27 \mathrm{~b}$ & $31.35 \mathrm{c}$ & $40.74 \mathrm{~b}$ & $13.83 \mathrm{~b}$ & $40.41 \mathrm{c}$ & $54.19 \mathrm{c}$ \\
\hline CV-08 & $8.30 \mathrm{c}$ & $29.97 \mathrm{c}$ & $38.33 \mathrm{c}$ & $10.27 \mathrm{~d}$ & $35.70 \mathrm{~d}$ & $45.97 \mathrm{e}$ \\
\hline CV-09 & $11.42 \mathrm{a}$ & $38.63 \mathrm{a}$ & $50.05 \mathrm{a}$ & $15.94 \mathrm{a}$ & $46.60 \mathrm{a}$ & $62.41 \mathrm{a}$ \\
\hline CV-10 & $5.47 \mathrm{~d}$ & $26.33 \mathrm{e}$ & $29.37 \mathrm{f}$ & $7.53 \mathrm{f}$ & $31.22 \mathrm{f}$ & $41.20 \mathrm{~g}$ \\
\hline CV-11 & $1.73 \mathrm{e}$ & $18.93 \mathrm{~g}$ & $20.59 \mathrm{~h}$ & $12.20 \mathrm{c}$ & $38.77 \mathrm{c}$ & $51.07 \mathrm{~d}$ \\
\hline $\mathrm{CV}-12$ & $2.43 \mathrm{e}$ & $23.65 \mathrm{f}$ & $26.07 \mathrm{~g}$ & $5.97 \mathrm{~g}$ & $26.54 \mathrm{~g}$ & $32.51 \mathrm{~h}$ \\
\hline \multirow[t]{2}{*}{ CV-13 } & $4.40 \mathrm{~d}$ & $24.91 \mathrm{e}$ & $29.37 \mathrm{f}$ & $8.59 \mathrm{e}$ & $40.66 \mathrm{c}$ & $50.23 \mathrm{~d}$ \\
\hline & \multicolumn{3}{|c|}{$100 \%$ of $\mathrm{P}_{2} \mathrm{O}_{5}$} & \multicolumn{3}{|c|}{$150 \%$ of $\mathrm{P}_{2} \mathrm{O}_{5}$} \\
\hline CV-01 & $15.14 \mathrm{~d}$ & $51.42 \mathrm{~b}$ & $66.51 \mathrm{c}$ & $16.10 \mathrm{~g}$ & $63.44 \mathrm{c}$ & $79.65 \mathrm{f}$ \\
\hline $\mathrm{CV}-02$ & $17.58 \mathrm{c}$ & $40.51 \mathrm{e}$ & $58.09 \mathrm{f}$ & $19.40 \mathrm{f}$ & $52.06 \mathrm{e}$ & $71.43 \mathrm{~g}$ \\
\hline $\mathrm{CV}-03$ & $19.67 \mathrm{~b}$ & $49.62 \mathrm{c}$ & $69.15 \mathrm{~b}$ & $29.91 \mathrm{a}$ & $62.30 \mathrm{c}$ & $92.03 \mathrm{c}$ \\
\hline CV-04 & $14.67 \mathrm{~d}$ & $42.88 \mathrm{e}$ & $57.25 \mathrm{f}$ & $16.44 \mathrm{~g}$ & $63.69 \mathrm{c}$ & $80.13 \mathrm{f}$ \\
\hline CV-05 & $21.37 \mathrm{a}$ & $51.21 \mathrm{~b}$ & $72.62 \mathrm{a}$ & $28.66 \mathrm{~b}$ & $65.34 \mathrm{~b}$ & $93.62 \mathrm{~b}$ \\
\hline CV-06 & $17.88 \mathrm{c}$ & $55.68 \mathrm{a}$ & $73.56 \mathrm{a}$ & $20.78 \mathrm{e}$ & $69.61 \mathrm{a}$ & $90.59 \mathrm{c}$ \\
\hline CV-07 & $20.15 \mathrm{~b}$ & $52.05 \mathrm{~b}$ & $72.27 \mathrm{a}$ & $30.04 \mathrm{a}$ & $66.72 \mathrm{~b}$ & $96.97 \mathrm{a}$ \\
\hline CV-08 & $23.70 \mathrm{a}$ & $48.69 \mathrm{c}$ & $72.39 \mathrm{a}$ & $24.45 \mathrm{~d}$ & $65.49 \mathrm{~b}$ & $90.03 \mathrm{c}$ \\
\hline CV-09 & $20.45 \mathrm{a}$ & $52.41 \mathrm{~b}$ & $72.89 \mathrm{a}$ & $26.71 \mathrm{c}$ & $60.93 \mathrm{~d}$ & $87.50 \mathrm{~d}$ \\
\hline CV-10 & $13.25 \mathrm{e}$ & $52.43 \mathrm{~b}$ & $65.69 \mathrm{c}$ & $22.33 \mathrm{e}$ & $63.25 \mathrm{c}$ & $85.47 \mathrm{e}$ \\
\hline CV-11 & $14.64 \mathrm{~d}$ & $46.15 \mathrm{~d}$ & $60.80 \mathrm{e}$ & $25.69 \mathrm{c}$ & $62.71 \mathrm{c}$ & $88.12 \mathrm{~d}$ \\
\hline $\mathrm{CV}-12$ & $11.72 \mathrm{f}$ & $50.42 \mathrm{c}$ & $62.14 \mathrm{~d}$ & $19.48 \mathrm{f}$ & $64.17 \mathrm{c}$ & $83.51 \mathrm{e}$ \\
\hline CV-13 & $13.00 \mathrm{e}$ & $50.65 \mathrm{c}$ & $63.55 \mathrm{~d}$ & $23.80 \mathrm{~d}$ & $60.56 \mathrm{~d}$ & $84.46 \mathrm{e}$ \\
\hline
\end{tabular}

Note: Means followed by the same letter in the row do not differ from each other by the Scott-Knott test $(\mathrm{p} \leq 0.05) .{ }^{1}$ Dry matter of roots $\left(\mathrm{g}\right.$ plant $\left.{ }^{-1}\right) \dot{ }^{2}$ Dry matter of aerial part $\left(\mathrm{g}\right.$ plant $\left.\mathrm{t}^{-1}\right) ;{ }^{3}$ Total dry matter $\left(\mathrm{g}\right.$ plant $\left.\mathrm{t}^{-1}\right)$.

The CV-09 clone showed high capacity for P accumulation, justified by its allocation in the higher average group of P content (PR, PAP and TP) at all levels of phosphorus fertilization studied. Also noteworthy are the clones CV-03 and CV-05 at levels of 100 and $150 \%$ of the recommended $\mathrm{P}_{2} \mathrm{O}_{5}$. The clone CV-12 showed reduced $\mathrm{P}$ content in their vegetative parts (PR, PAP and TP), for all levels of phosphorus applied on the soil (Table 3). 
In studies of corn cultivars under different levels of phosphorus, cultivated in nutrient solution, Machado et al. (2001) found out that the total content of $P$ was different for the cultivars within each level of phosphorus, showing a similar behavior to that observed in the clones of conilon coffee. According to Malavolta (2006), providing different levels of phosphorus, the total content of $\mathrm{P}$ in the plant, too, can be different due to the difference in genetic capacity of each individual upon its mineral nutrition.

Table 3. Means of phosphorus content of the clones of conilon coffee that compose the clonal cultivar "Vitória Incaper 8142" for each level of phosphorus fertilization $\left(0,50,100\right.$ and $150 \%$ of the recommended level of $\mathrm{P}_{2} \mathrm{O}_{5}$ for the culture)

\begin{tabular}{|c|c|c|c|c|c|c|}
\hline \multirow{2}{*}{ Clone } & $\overline{\mathrm{PR}^{1}}$ & $\mathrm{PAP}^{2}$ & $\mathrm{TP}^{3}$ & $\mathrm{PR}^{1}$ & $\overline{\text { PAP }^{2}}$ & $\mathrm{TP}^{3}$ \\
\hline & \multicolumn{3}{|c|}{$0 \%$ of $\mathrm{P}_{2} \mathrm{O}_{5}$} & \multicolumn{3}{|c|}{$50 \%$ of $\mathrm{P}_{2} \mathrm{O}_{5}$} \\
\hline $\mathrm{CV}-01$ & $6.45 \mathrm{c}$ & $35.54 \mathrm{~b}$ & $42.00 \mathrm{~b}$ & $14.89 \mathrm{c}$ & $58.41 \mathrm{c}$ & $73.31 \mathrm{~b}$ \\
\hline $\mathrm{CV}-02$ & $8.16 \mathrm{~b}$ & $32.46 \mathrm{~b}$ & $40.62 \mathrm{~b}$ & $17.19 \mathrm{~b}$ & $53.05 \mathrm{~d}$ & $70.24 \mathrm{~b}$ \\
\hline $\mathrm{CV}-03$ & $6.31 \mathrm{c}$ & $35.97 \mathrm{~b}$ & $42.29 \mathrm{~b}$ & $12.97 \mathrm{~d}$ & $57.33 \mathrm{c}$ & $70.30 \mathrm{~b}$ \\
\hline CV-04 & $6.18 \mathrm{c}$ & $33.47 \mathrm{~b}$ & $39.66 \mathrm{~b}$ & $11.02 \mathrm{e}$ & $47.73 \mathrm{e}$ & $58.76 \mathrm{~d}$ \\
\hline $\mathrm{CV}-05$ & $9.17 \mathrm{~b}$ & $29.41 \mathrm{c}$ & $38.59 \mathrm{~b}$ & $10.65 \mathrm{e}$ & $52.60 \mathrm{~d}$ & $63.26 \mathrm{c}$ \\
\hline CV-06 & $0.89 \mathrm{e}$ & $27.70 \mathrm{c}$ & $28.59 \mathrm{~d}$ & $13.60 \mathrm{~d}$ & $60.18 \mathrm{~b}$ & $73.78 \mathrm{~b}$ \\
\hline CV-07 & $9.52 \mathrm{~b}$ & $34.01 \mathrm{~b}$ & $43.54 \mathrm{~b}$ & $14.84 \mathrm{c}$ & $56.27 \mathrm{c}$ & $71.11 \mathrm{~b}$ \\
\hline $\mathrm{CV}-08$ & $9.27 \mathrm{~b}$ & $31.40 \mathrm{~b}$ & $40.67 \mathrm{~b}$ & $11.17 \mathrm{e}$ & $37.62 \mathrm{~g}$ & $48.80 \mathrm{e}$ \\
\hline CV-09 & $11.01 \mathrm{a}$ & $40.52 \mathrm{a}$ & $51.54 \mathrm{a}$ & $20.71 \mathrm{a}$ & $70.63 \mathrm{a}$ & $91.34 \mathrm{a}$ \\
\hline $\mathrm{CV}-10$ & $1.97 \mathrm{e}$ & $24.32 \mathrm{~d}$ & $26.29 \mathrm{~d}$ & $7.00 \mathrm{~g}$ & $40.83 \mathrm{f}$ & $47.83 \mathrm{e}$ \\
\hline$C V-11$ & $1.37 \mathrm{e}$ & $20.11 \mathrm{e}$ & $25.49 \mathrm{~d}$ & $13.22 \mathrm{~d}$ & $59.33 \mathrm{~b}$ & $72.56 \mathrm{~b}$ \\
\hline $\mathrm{CV}-12$ & $0.89 \mathrm{e}$ & $25.32 \mathrm{~d}$ & $26.22 \mathrm{~d}$ & $6.30 \mathrm{~g}$ & $34.96 \mathrm{~g}$ & $41.27 \mathrm{f}$ \\
\hline \multirow[t]{2}{*}{$C V-13$} & $4.07 \mathrm{~d}$ & $29.59 \mathrm{c}$ & $37.67 \mathrm{c}$ & $8.82 \mathrm{f}$ & $60.91 \mathrm{~b}$ & $69.73 \mathrm{~b}$ \\
\hline & \multicolumn{3}{|c|}{$100 \%$ of $\mathrm{P}_{2} \mathrm{O}_{5}$} & \multicolumn{3}{|c|}{$150 \%$ of $\mathrm{P}_{2} \mathrm{O}_{5}$} \\
\hline $\mathrm{CV}-01$ & $17.66 \mathrm{~d}$ & $77.51 \mathrm{~d}$ & $95.18 \mathrm{e}$ & $20.93 \mathrm{~g}$ & $102.43 \mathrm{e}$ & $123.37 \mathrm{f}$ \\
\hline $\mathrm{CV}-02$ & $24.01 \mathrm{~b}$ & $62.90 \mathrm{f}$ & $86.92 \mathrm{f}$ & $29.35 \mathrm{e}$ & $89.80 \mathrm{f}$ & $119.15 \mathrm{~g}$ \\
\hline $\mathrm{CV}-03$ & $23.82 \mathrm{~b}$ & $109.84 \mathrm{a}$ & $133.67 \mathrm{a}$ & $39.50 \mathrm{~b}$ & $118.39 \mathrm{c}$ & $157.89 \mathrm{~b}$ \\
\hline CV-04 & $17.14 \mathrm{~d}$ & $69.98 \mathrm{e}$ & $87.13 \mathrm{f}$ & $23.19 \mathrm{f}$ & $113.92 \mathrm{~d}$ & $137.11 \mathrm{e}$ \\
\hline CV-05 & $29.09 \mathrm{a}$ & $83.04 \mathrm{c}$ & $112.13 \mathrm{~b}$ & $45.34 \mathrm{a}$ & $120.64 \mathrm{~b}$ & $165.99 \mathrm{a}$ \\
\hline CV-06 & $16.88 \mathrm{~d}$ & $89.24 \mathrm{~b}$ & $106.13 \mathrm{c}$ & $21.43 \mathrm{~g}$ & $126.67 \mathrm{a}$ & $148.10 \mathrm{~d}$ \\
\hline $\mathrm{CV}-07$ & $24.43 \mathrm{~b}$ & $83.93 \mathrm{c}$ & $108.37 \mathrm{c}$ & $43.81 \mathrm{a}$ & $117.74 \mathrm{c}$ & $161.55 \mathrm{~b}$ \\
\hline CV-08 & $25.29 \mathrm{~b}$ & $76.65 \mathrm{~d}$ & $101.95 \mathrm{~d}$ & $30.37 \mathrm{~d}$ & $105.43 \mathrm{e}$ & $135.80 \mathrm{e}$ \\
\hline CV-09 & $28.62 \mathrm{a}$ & $79.36 \mathrm{~d}$ & $107.98 \mathrm{c}$ & $40.14 \mathrm{~b}$ & $120.21 \mathrm{~b}$ & $160.36 \mathrm{~b}$ \\
\hline $\mathrm{CV}-10$ & $16.04 \mathrm{~d}$ & $91.04 \mathrm{~b}$ & $107.08 \mathrm{c}$ & $28.69 \mathrm{e}$ & $120.96 \mathrm{~b}$ & $149.66 \mathrm{c}$ \\
\hline CV-11 & $19.55 \mathrm{c}$ & $76.10 \mathrm{~d}$ & $95.65 \mathrm{e}$ & $36.91 \mathrm{c}$ & $114.84 \mathrm{~d}$ & $151.76 \mathrm{c}$ \\
\hline $\mathrm{CV}-12$ & $12.91 \mathrm{f}$ & $72.68 \mathrm{e}$ & $85.59 \mathrm{f}$ & $25.00 \mathrm{f}$ & $120.32 \mathrm{~b}$ & $145.33 \mathrm{~d}$ \\
\hline $\mathrm{CV}-13$ & $14.70 \mathrm{e}$ & $81.29 \mathrm{c}$ & $95.99 \mathrm{e}$ & $31.30 \mathrm{~d}$ & $116.00 \mathrm{~d}$ & $147.31 \mathrm{~d}$ \\
\hline
\end{tabular}

Note: Means followed by the same letter in the row do not differ from each other by the Scott-Knott test $(\mathrm{p} \leq 0.05) .{ }^{1}$ Phosphorus content in the roots $\left(\mathrm{g} \mathrm{plant}^{-1}\right) ;{ }^{2}$ Phosphorus content in the aerial part $\left(\mathrm{g}\right.$ plant $\left.{ }^{-1}\right) ;{ }^{3}$ Total phosphorus content $\left(\mathrm{g} \mathrm{plant}^{-1}\right)$.

In Table 4, it can be verified that all analyzed clones of conilon coffee present, for the variables of dry matter (DMR, DMAP and TDM) and P content (PR, PAP and TP), fit to a linear crescent model based on the 
administered levels of phosphorus fertilization, checking that the maximum values observed for the variables are presented at the level of $150 \%$ of the recommended $\mathrm{P}_{2} \mathrm{O}_{5}$ for the culture.

Table 4. Regression equations, with their respective determination coefficients $\left(\mathrm{R}^{2}\right)$, for dry matter and phosphorus content of the clones of conilon coffee that compose the cultivar "Vitória Incaper 8142", in function of the levels of phosphorus $\left(0,50,100\right.$ and $150 \%$ of the recommended level of $\mathrm{P}_{2} \mathrm{O}_{5}$ for the culture)

\begin{tabular}{|c|c|c|c|c|c|c|}
\hline Clone & Variable & Equation & $\overline{\mathrm{R}^{2}}$ & Variable & Equation & $\mathrm{R}^{2}$ \\
\hline \multirow{3}{*}{ CV-01 } & $\mathrm{DMR}^{1}$ & $\hat{\mathrm{Y}}=0.0540 * \mathrm{P}+9.557$ & 0.86 & $\mathrm{PR}^{4}$ & $\hat{\mathrm{Y}}=0.092 * \mathrm{P}+8.058$ & 0.92 \\
\hline & DMAP $^{2}$ & $\hat{\mathrm{Y}}=0.1925 * \mathrm{P}+33.605$ & 0.99 & PAP $^{5}$ & $\hat{\mathrm{Y}}=0.439 * \mathrm{P}+35.50$ & 0.99 \\
\hline & $\mathrm{TDM}^{3}$ & $\hat{\mathrm{Y}}=0.2444 * \mathrm{P}+43.316$ & 0.97 & $\mathrm{TP}^{6}$ & $\hat{\mathrm{Y}}=0.531 * \mathrm{P}+43.57$ & 0.99 \\
\hline \multirow{3}{*}{ CV-02 } & $\mathrm{DMR}^{1}$ & $\hat{\mathrm{Y}}=0.0826^{*} \mathrm{P}+8.157$ & 0.85 & $\mathrm{PR}^{4}$ & $\hat{\mathrm{Y}}=0.140 * \mathrm{P}+9.123$ & 0.98 \\
\hline & DMAP $^{2}$ & $\hat{\mathrm{Y}}=0.1814 * \mathrm{P}+24.57$ & 0.95 & PAP $^{5}$ & $\hat{\mathrm{Y}}=0.363^{*} \mathrm{P}+32.27$ & 0.97 \\
\hline & $\mathrm{TDM}^{3}$ & $\hat{\mathrm{Y}}=0.255^{*} \mathrm{P}+33.733$ & 0.93 & $\mathrm{TP}^{6}$ & $\hat{\mathrm{Y}}=0.504 * \mathrm{P}+41.39$ & 0.98 \\
\hline \multirow{3}{*}{ CV-03 } & $\mathrm{DMR}^{1}$ & $\hat{\mathrm{Y}}=0.1588 * \mathrm{P}+4.914$ & 0.98 & $\mathrm{PR}^{4}$ & $\hat{\mathrm{Y}}=0.220 * \mathrm{P}+4.091$ & 0.96 \\
\hline & DMAP $^{2}$ & $\hat{\mathrm{Y}}=0.2294 * \mathrm{P}+26.930$ & 0.98 & $\mathrm{PAP}^{5}$ & $\hat{\mathrm{Y}}=0.599 * \mathrm{P}+35.41$ & 0.93 \\
\hline & $\mathrm{TDM}^{3}$ & $\hat{\mathrm{Y}}=0.3867 * \mathrm{P}+31.917$ & 0.98 & $\mathrm{TP}^{6}$ & $\hat{\mathrm{Y}}=0.820 * \mathrm{P}+39.51$ & 0.96 \\
\hline \multirow{3}{*}{ CV-04 } & $\mathrm{DMR}^{1}$ & $\hat{\mathrm{Y}}=0.1588^{*} \mathrm{P}+4.914$ & 0.98 & $\mathrm{PR}^{4}$ & $\hat{\mathrm{Y}}=0.114 * \mathrm{P}+5.818$ & 0.99 \\
\hline & DMAP $^{2}$ & $\hat{\mathrm{Y}}=0.2416^{*} \mathrm{P}+23.408$ & 0.92 & PAP $^{5}$ & $\hat{\mathrm{Y}}=0.527 * \mathrm{P}+26.73$ & 0.93 \\
\hline & $\mathrm{TDM}^{3}$ & $\hat{\mathrm{Y}}=0.3171 * \mathrm{P}+29.132$ & 0.96 & $\mathrm{TP}^{6}$ & $\hat{\mathrm{Y}}=0.527^{*} \mathrm{P}+26.73$ & 0.93 \\
\hline \multirow{3}{*}{ CV-05 } & $\mathrm{DMR}^{1}$ & $\hat{\mathrm{Y}}=0.1351 * \mathrm{P}+7.478$ & 0.92 & $\mathrm{PR}^{4}$ & $\hat{\mathrm{Y}}=0.253 * \mathrm{P}+4.525$ & 0.91 \\
\hline & DMAP $^{2}$ & $\hat{\mathrm{Y}}=0.2597 * \mathrm{P}+25.156$ & 0.97 & $\mathrm{PAP}^{5}$ & $\hat{\mathrm{Y}}=0.608^{*} \mathrm{P}+25.80$ & 0.98 \\
\hline & $\mathrm{TDM}^{3}$ & $\hat{\mathrm{Y}}=0.3929 * \mathrm{P}+32.676$ & 0.95 & $\mathrm{TP}^{6}$ & $\hat{\mathrm{Y}}=0.862 * \mathrm{P}+30.33$ & 0.97 \\
\hline \multirow{3}{*}{ CV-06 } & $\mathrm{DMR}^{1}$ & $\hat{\mathrm{Y}}=0.0958 * \mathrm{P}+7.693$ & 0.89 & $\mathrm{PR}^{4}$ & $\hat{\mathrm{Y}}=0.129 * \mathrm{P}+3.470$ & 0.90 \\
\hline & DMAP $^{2}$ & $\hat{\mathrm{Y}}=0.2832 * \mathrm{P}+27.643$ & 0.99 & $\mathrm{PAP}^{5}$ & $\hat{\mathrm{Y}}=0.651 * \mathrm{P}+27.05$ & 0.99 \\
\hline & $\mathrm{TDM}^{3}$ & $\hat{\mathrm{Y}}=0.3805^{*} \mathrm{P}+35.246$ & 0.98 & $\mathrm{TP}^{6}$ & $\hat{\mathrm{Y}}=0.781 * \mathrm{P}+30.52$ & 0.99 \\
\hline \multirow{3}{*}{ CV-07 } & $\mathrm{DMR}^{1}$ & $\hat{\mathrm{Y}}=0.1374 * \mathrm{P}+8.005$ & 0.97 & $\mathrm{PR}^{4}$ & $\hat{\mathrm{Y}}=0.224 * \mathrm{P}+6.289$ & 0.92 \\
\hline & $\mathrm{DMAP}^{2}$ & $\hat{\mathrm{Y}}=0.2355^{*} \mathrm{P}+29.969$ & 0.98 & $\mathrm{PAP}^{5}$ & $\hat{\mathrm{Y}}=0.557 * \mathrm{P}+31.16$ & 0.99 \\
\hline & $\mathrm{TDM}^{3}$ & $\hat{\mathrm{Y}}=0.3736 * \mathrm{P}+38.029$ & 0.98 & $\mathrm{TP}^{6}$ & $\hat{\mathrm{Y}}=0.557 * \mathrm{P}+31.16$ & 0.99 \\
\hline \multirow{3}{*}{ CV-08 } & $\mathrm{DMR}^{1}$ & $\hat{\mathrm{Y}}=0.1234 * \mathrm{P}+7.438$ & 0.86 & $\mathrm{PR}^{4}$ & $\hat{\mathrm{Y}}=0.154 * \mathrm{P}+7.417$ & 0.92 \\
\hline & $\mathrm{DMAP}^{2}$ & $\hat{\mathrm{Y}}=0.2391 * \mathrm{P}+27.036$ & 0.95 & $\mathrm{PAP}^{5}$ & $\hat{\mathrm{Y}}=0.363 * \mathrm{P}+34.45$ & 0.96 \\
\hline & $\mathrm{TDM}^{3}$ & $\hat{\mathrm{Y}}=0.3630 * \mathrm{P}+34.457$ & 0.96 & $\mathrm{TP}^{6}$ & $\hat{\mathrm{Y}}=0.363 * \mathrm{P}+34.45$ & 0.96 \\
\hline \multirow{3}{*}{ CV-09 } & $\mathrm{DMR}^{1}$ & $\hat{\mathrm{Y}}=0.1008 * \mathrm{P}+11.072$ & 0.99 & $\mathrm{PR}^{4}$ & $\hat{\mathrm{Y}}=0.190 * \mathrm{P}+10.82$ & 0.99 \\
\hline & $\mathrm{DMAP}^{2}$ & $\hat{\mathrm{Y}}=0.1455^{*} \mathrm{P}+38.735$ & 0.99 & $\mathrm{PAP}^{5}$ & $\hat{\mathrm{Y}}=0.495^{*} \mathrm{P}+40.51$ & 0.94 \\
\hline & $\mathrm{TDM}^{3}$ & $\hat{\mathrm{Y}}=0.2457 * \mathrm{P}+49.788$ & 0.99 & $\mathrm{TP}^{6}$ & $\hat{\mathrm{Y}}=0.495^{*} \mathrm{P}+40.51$ & 0.94 \\
\hline \multirow{3}{*}{ CV-10 } & $\mathrm{DMR}^{1}$ & $\hat{\mathrm{Y}}=0.1125 * \mathrm{P}+3.719$ & 0.92 & $\mathrm{PR}^{4}$ & $\hat{\mathrm{Y}}=0.178 * \mathrm{P}+0.047$ & 0.96 \\
\hline & $\mathrm{DMAP}^{2}$ & $\hat{\mathrm{Y}}=0.2639 * \mathrm{P}+23.514$ & 0.95 & $\mathrm{PAP}^{5}$ & $\hat{\mathrm{Y}}=0.680 * \mathrm{P}+18.26$ & 0.96 \\
\hline & $\mathrm{TDM}^{3}$ & $\hat{\mathrm{Y}}=0.3855^{*} \mathrm{P}+26.521$ & 0.98 & $\mathrm{TP}^{6}$ & $\hat{\mathrm{Y}}=0.858 * \mathrm{P}+18.32$ & 0.97 \\
\hline \multirow{3}{*}{ CV-11 } & $\mathrm{DMR}^{1}$ & $\hat{\mathrm{Y}}=0.1487 * \mathrm{P}+2.408$ & 0.95 & $\mathrm{PR}^{4}$ & $\hat{\mathrm{Y}}=0.225^{*} \mathrm{P}+0.827$ & 0.96 \\
\hline & $\mathrm{DMAP}^{2}$ & $\hat{\mathrm{Y}}=0.2775^{*} \mathrm{P}+20.836$ & 0.97 & PAP $^{5}$ & $\hat{\mathrm{Y}}=0.601 * \mathrm{P}+22.45$ & 0.97 \\
\hline & $\mathrm{TDM}^{3}$ & $\hat{\mathrm{Y}}=0.4246^{*} \mathrm{P}+23.302$ & 0.96 & $\mathrm{TP}^{6}$ & $\hat{\mathrm{Y}}=0.827^{*} \mathrm{P}+23.28$ & 0.97 \\
\hline \multirow{3}{*}{$\mathrm{CV}-12$} & $\mathrm{DMR}^{1}$ & $\hat{\mathrm{Y}}=0.1139 * \mathrm{P}+1.358$ & 0.97 & $\mathrm{PR}^{4}$ & $\hat{\mathrm{Y}}=0.157 * \mathrm{P}-0.557$ & 0.96 \\
\hline & DMAP $^{2}$ & $\hat{\mathrm{Y}}=0.2909 * \mathrm{P}+19.381$ & 0.93 & $\mathrm{PAP}^{5}$ & $\hat{\mathrm{Y}}=0.645^{*} \mathrm{P}+14.91$ & 0.93 \\
\hline & $\mathrm{TDM}^{3}$ & $\hat{\mathrm{Y}}=0.4039 * \mathrm{P}+20.768$ & 0.95 & $\mathrm{TP}^{6}$ & $\hat{\mathrm{Y}}=0.803 * \mathrm{P}+14.35$ & 0.94 \\
\hline \multirow{3}{*}{ CV-13 } & $\mathrm{DMR}^{1}$ & $\hat{\mathrm{Y}}=0.1249 * \mathrm{P}+3.100$ & 0.93 & $\mathrm{PR}^{4}$ & $\hat{\mathrm{Y}}=0.175^{*} \mathrm{P}+1.593$ & 0.90 \\
\hline & $\mathrm{DMAP}^{2}$ & $\hat{\mathrm{Y}}=0.2339 * \mathrm{P}+26.656$ & 0.98 & $\mathrm{PAP}^{5}$ & $\hat{\mathrm{Y}}=0.559 * \mathrm{P}+30.00$ & 0.99 \\
\hline & $\mathrm{TDM}^{3}$ & $\hat{\mathrm{Y}}=0.3572 * \mathrm{P}+30.118$ & 0.99 & $\mathrm{TP}^{6}$ & $\hat{\mathrm{Y}}=0.734 * \mathrm{P}+31.60$ & 0.98 \\
\hline
\end{tabular}

Note: ${ }^{*}$ Significative by the $\mathrm{t}$ test, at $5 \%$ of probability. ${ }^{1}$ Dry matter of roots $\left(\mathrm{g}\right.$ plant $\left.{ }^{-1}\right) ;{ }^{2}$ Dry matter of aerial part (g plant $\left.{ }^{-1}\right) ;{ }^{3}$ Total dry matter $\left(\mathrm{g}\right.$ plant $\left.{ }^{-1}\right) .{ }^{4}$ Phosphorus content in the roots $\left(\mathrm{g}\right.$ plant $\left.{ }^{-1}\right) ;{ }^{5}$ Phosphorus content in the aerial part $\left(\mathrm{g}\right.$ plant $\left.{ }^{-1}\right) ;{ }^{6}$ Total phosphorus content $\left(\mathrm{g}\right.$ plant $\left.{ }^{-1}\right)$. 
There was a larger vegetative growth, observed by the significant increase in dry weight (Table 4), due to the greater availability of $\mathrm{P}$ in the soil as a function of the increasing levels of $\mathrm{P}_{2} \mathrm{O}_{5}$ applied. Analyzing the growth and photosynthesis of young plants of arabica coffee, Silva et al. (2010) found that the increased availability of phosphorus in the soil promoted greater plant growth. These authors attributed the higher accumulation of dry matter by the high photosynthetic performance, influenced by greater stomatal opening, greater biochemical and photochemical activity of the coffee seedlings, which have thus increased availability of energy as carbohydrates for growth and subsequent conversion into dry matter.

In this work, despite not been evaluated metabolic parameters, it is assumed that the relationship presented by Silva et al. (2010), too, can justify the dry matter values of the conilon coffee in function of the levels of fertilization with $\mathrm{P}_{2} \mathrm{O}_{5}$ used in this study.

The linear increase of $\mathrm{P}$ content (Table 4) can be justified as result of the processes of absorption and translocation, and also photosynthesis which have been maximized as the levels of $\mathrm{P}_{2} \mathrm{O}_{5}$ were increased (Malavolta, 2006). The phosphorus content in the vegetative parts rises as the availability of $\mathrm{P}$ in soil grows (Novais \& Smyth, 1999), given to the difficulty of fully saturate the soil with phosphorus, due to the peculiarities of Brazilian soils (Novais et al., 2007).

\subsection{Nutritional Efficiency of $P$ of Clones of Conilon Coffee}

As in the other studied variables, the clones showed different nutritional efficiencies (AE, UE and TE), as evidenced by the different composition settings of group of means at each level of $\mathrm{P}_{2} \mathrm{O}_{5}$ studied (Table 5).

Table 5. Means of efficiencies for phosphorus absorption, translocation and utilization in the clones that compose the cultivar of conilon coffee " Vitória Incaper 8142" for each level of phosphorus $(0,50,100$ and 150\% of the recommended level of $\mathrm{P}_{2} \mathrm{O}_{5}$ for the culture)

\begin{tabular}{|c|c|c|c|c|c|c|}
\hline \multirow{2}{*}{ Clone } & $\mathrm{AE}^{1}$ & $\mathrm{TE}^{2}$ & $\mathrm{UE}^{3}$ & $\mathrm{AE}^{\mathrm{I}}$ & $\mathrm{TE}^{2}$ & $\mathrm{UE}^{3}$ \\
\hline & \multicolumn{3}{|c|}{$0 \%$ of $\mathrm{P}_{2} \mathrm{O}_{5}$} & \multicolumn{3}{|c|}{$50 \%$ of $\mathrm{P}_{2} \mathrm{O}_{5}$} \\
\hline CV-01 & $5.72 \mathrm{~d}$ & $84.60 \mathrm{~d}$ & $40.82 \mathrm{~b}$ & $4.64 \mathrm{~b}$ & $79.66 \mathrm{~d}$ & 47.50 \\
\hline$J-02$ & $4 \mathrm{~d}$ & $96.18 \mathrm{a}$ & $20 \mathrm{i}$ & $24 \mathrm{~b}$ & $75.52 \mathrm{e}$ & 36.45 \\
\hline$I_{-} 03$ & $0 \mathrm{c}$ & $85.08 \mathrm{~d}$ & $28.48 \mathrm{f}$ & $3 \mathrm{~b}$ & $81.59 \mathrm{c}$ & $32.47 \mathrm{~d}$ \\
\hline$T_{-0}$ & $25 \mathrm{c}$ & $84.42 \mathrm{~d}$ & $26.46 \mathrm{~g}$ & $38 \mathrm{a}$ & $81.23 \mathrm{c}$ & $29.86 \mathrm{e}$ \\
\hline 0 & $8 \mathrm{~d}$ & $76.19 \mathrm{f}$ & $36.20 \mathrm{~d}$ & $93 \mathrm{~b}$ & $83.16 \mathrm{c}$ & $31.91 \mathrm{~d}$ \\
\hline-06 & $1 \mathrm{~d}$ & $96.86 \mathrm{a}$ & $35.96 \mathrm{~d}$ & $80 \mathrm{~b}$ & $81.55 \mathrm{c}$ & $47.55 \mathrm{a}$ \\
\hline $\mathrm{C}$ & $33 \mathrm{~d}$ & $78.07 \mathrm{e}$ & $38.13 \mathrm{c}$ & $5.17 \mathrm{~b}$ & $79.12 \mathrm{~d}$ & $41.29 \mathrm{~b}$ \\
\hline$J-08$ & $37 \mathrm{~d}$ & $77.19 \mathrm{f}$ & $36.13 \mathrm{~d}$ & $75 \mathrm{~b}$ & $77.09 \mathrm{e}$ & $43.45 \mathrm{~b}$ \\
\hline$J-0$ & $1 \mathrm{~d}$ & $78.62 \mathrm{e}$ & $48.60 \mathrm{a}$ & $5.78 \mathrm{~b}$ & $77.33 \mathrm{e}$ & $42.64 \mathrm{~b}$ \\
\hline$C$ & $30 \mathrm{~d}$ & $92.49 \mathrm{~b}$ & $32.81 \mathrm{e}$ & $6.35 \mathrm{a}$ & $85.33 \mathrm{~b}$ & $35.49 \mathrm{c}$ \\
\hline C & $15 \mathrm{a}$ & $93.61 \mathrm{~b}$ & $19.73 \mathrm{~h}$ & $91 \mathrm{~b}$ & $81.78 \mathrm{c}$ & $35.95 \mathrm{c}$ \\
\hline & $32 \mathrm{~b}$ & $96.56 \mathrm{a}$ & $25.92 \mathrm{~g}$ & $.07 \mathrm{a}$ & $84.77 \mathrm{~b}$ & $25.61 \mathrm{f}$ \\
\hline \multirow[t]{2}{*}{ CV-1. } & $5 \mathrm{c}$ & $87.89 \mathrm{c}$ & $25.62 \mathrm{~g}$ & $13 \mathrm{a}$ & $87.34 \mathrm{a}$ & 36.19 \\
\hline & \multicolumn{3}{|c|}{$100 \%$ of $\mathrm{P}_{2} \mathrm{O}_{5}$} & \multicolumn{3}{|c|}{$150 \%$ of $\mathrm{P}_{2} \mathrm{O}_{5}$} \\
\hline $\mathrm{CV}-0$ & $31 \mathrm{a}$ & $81.44 \mathrm{~b}$ & $46.48 \mathrm{~b}$ & $7.66 \mathrm{a}$ & $83.03 \mathrm{~b}$ & $51.42 \mathrm{c}$ \\
\hline $\mathrm{CV}$ & $8 \mathrm{~b}$ & $72.40 \mathrm{f}$ & $38.82 \mathrm{~d}$ & $6.15 \mathrm{~b}$ & $75.37 \mathrm{e}$ & $42.82 \mathrm{e}$ \\
\hline $\mathrm{CV}$ & $35 \mathrm{a}$ & $82.17 \mathrm{~b}$ & $35.77 \mathrm{e}$ & $5.31 \mathrm{~b}$ & $74.97 \mathrm{e}$ & $53.64 \mathrm{~b}$ \\
\hline Cl & $9 a$ & $80.34 \mathrm{c}$ & $37.62 \mathrm{~d}$ & $8.34 \mathrm{a}$ & $83.08 \mathrm{~b}$ & $46.83 \mathrm{~d}$ \\
\hline CV & $3 \mathrm{~b}$ & $74.05 \mathrm{f}$ & $47.04 \mathrm{~b}$ & $5.87 \mathrm{~b}$ & $72.67 \mathrm{f}$ & $52.80 \mathrm{~b}$ \\
\hline C & $6 \mathrm{a}$ & $84.09 \mathrm{a}$ & $50.07 \mathrm{a}$ & $7.06 \mathrm{a}$ & $85.52 \mathrm{a}$ & $55.41 \mathrm{~b}$ \\
\hline C & $5 \mathrm{~b}$ & $77.42 \mathrm{~d}$ & $48.20 \mathrm{~b}$ & $5.34 \mathrm{~b}$ & $72.84 \mathrm{f}$ & $58.20 \mathrm{a}$ \\
\hline $\mathrm{CV}-\mathrm{C}$ & $0 \mathrm{~b}$ & $19 \mathrm{e}$ & $\mathrm{a}$ & $5.53 \mathrm{~b}$ & $77.62 \mathrm{~d}$ & $59.69 \mathrm{a}$ \\
\hline $\mathrm{C}$ & $7 \mathrm{~b}$ & $48 \mathrm{f}$ & $49.20 \mathrm{a}$ & $6.03 \mathrm{~b}$ & $74.96 \mathrm{e}$ & $47.75 \mathrm{~d}$ \\
\hline $\mathrm{C}$ & $8 \mathrm{a}$ & $.02 \mathrm{a}$ & $40.29 \mathrm{c}$ & $6.73 \mathrm{a}$ & $80.82 \mathrm{c}$ & $48.81 \mathrm{~d}$ \\
\hline $\mathrm{C}$ & $\mathrm{a}$ & $7 \mathrm{c}$ & $38.64 \mathrm{~d}$ & $5.97 \mathrm{~b}$ & $75.67 \mathrm{e}$ & $51.16 \mathrm{c}$ \\
\hline C) & & $.89 \mathrm{a}$ & $45.11 \mathrm{~b}$ & $7.51 \mathrm{a}$ & $82.79 \mathrm{~b}$ & $47.99 \mathrm{~d}$ \\
\hline & $7.45 \mathrm{a}$ & $84.68 \mathrm{a}$ & $42.07 \mathrm{c}$ & $6.16 \mathrm{~b}$ & $78.74 \mathrm{~d}$ & $48.43 \mathrm{~d}$ \\
\hline
\end{tabular}

Note: Means followed by the same letter in the row do not differ from each other by the Scott-Knott test $(\mathrm{p} \leq 0.05) .{ }^{1}$ Absorption efficiency $\left(\mathrm{mg} \mathrm{g}^{-1}\right) ;{ }^{2}$ Translocation efficiency $(\%) ;{ }^{3}$ Utilization efficiency $\left(\mathrm{g}^{2} \mathrm{mg}^{-1}\right)$. 
Table 6. Regression equations, with their respective determination coefficients $\left(\mathrm{R}^{2}\right)$, for of efficiencies for phosphorus absorption, translocation and utilization of the clones of conilon coffee that compose the cultivar "Vitória Incaper 8142", in function of the levels of phosphorus $(0,50,100$ and $150 \%$ of the recommended level of $\mathrm{P}_{2} \mathrm{O}_{5}$ for the culture)

\begin{tabular}{|c|c|c|c|}
\hline Clone & Variable & Equation & $\mathrm{R}^{2}$ \\
\hline & $\mathrm{AE}^{1}$ & $\hat{\mathrm{Y}}=0.0002 * \mathrm{P}^{2}-0.0214^{\mathrm{ns}} \mathrm{P}+5.5762$ & 0.90 \\
\hline \multirow[t]{3}{*}{ CV-01 } & $\mathrm{TE}^{2}$ & $\hat{\mathrm{Y}}=0.0007 * \mathrm{P}^{2}-0.1039 * \mathrm{P}+84.262$ & 0.82 \\
\hline & $\mathrm{UE}^{3}$ & $\hat{\mathrm{Y}}=0.0616^{*} \mathrm{P}+41.944$ & 0.82 \\
\hline & $\mathrm{AE}^{1}$ & $\hat{\mathrm{Y}}=0.0002 * \mathrm{P}^{2}-0.0306 * \mathrm{P}+6.1420$ & 0.94 \\
\hline \multirow[t]{3}{*}{ CV-02 } & $\mathrm{TE}^{2}$ & $\hat{\mathrm{Y}}=0.0024 * \mathrm{P}^{2}-0.4856 * \mathrm{P}+95.615$ & 0.98 \\
\hline & $\mathrm{UE}^{3}$ & $\hat{\mathrm{Y}}=0.1585^{*} \mathrm{P}+21.943$ & 0.80 \\
\hline & $\mathrm{AE}^{1}$ & $\hat{\mathrm{Y}}=\overline{\mathrm{Y}}=6.703$ & - \\
\hline \multirow[t]{3}{*}{ CV-03 } & $\mathrm{TE}^{2}$ & $\hat{\mathrm{Y}}=-0.0595 * \mathrm{P}+85.419$ & 0.81 \\
\hline & $\mathrm{UE}^{3}$ & $\hat{\mathrm{Y}}=0.1575^{*} \mathrm{P}+25.780$ & 0.83 \\
\hline & $\mathrm{AE}^{1}$ & $\hat{\mathrm{Y}}=0.0003 * \mathrm{P}^{2}-0.0407 * \mathrm{P}+7.3548$ & 0.93 \\
\hline \multirow[t]{3}{*}{ CV-04 } & $\mathrm{TE}^{2}$ & $\hat{\mathrm{Y}}=0.0006 * \mathrm{P}^{2}-0.0988 * \mathrm{P}+84.496$ & 0.99 \\
\hline & $\mathrm{UE}^{3}$ & $\hat{\mathrm{Y}}=0.1377 * \mathrm{P}+24.868$ & 0.96 \\
\hline & $\mathrm{AE}^{1}$ & $\hat{\mathrm{Y}}=\overline{\mathrm{Y}}=4.189$ & - \\
\hline \multirow[t]{3}{*}{ CV-05 } & $\mathrm{TE}^{2}$ & $\hat{\mathrm{Y}}=-0.0008 * \mathrm{P}^{2}+0.0859 * \mathrm{P}+77.386$ & 0.56 \\
\hline & $\mathrm{UE}^{3}$ & $\hat{\mathrm{Y}}=0.1298 * \mathrm{P}+32.254$ & 0.76 \\
\hline & $\mathrm{AE}^{1}$ & $\hat{\mathrm{Y}}=\overline{\mathrm{Y}}=4.915$ & \\
\hline \multirow[t]{3}{*}{ CV-06 } & $\mathrm{TE}^{2}$ & $\hat{\mathrm{Y}}=0.0017 * \mathrm{P}^{2}-0.314 * \mathrm{P}+95.918$ & 0.86 \\
\hline & $\mathrm{UE}^{3}$ & $\hat{\mathrm{Y}}=0.1217 * \mathrm{P}+38.125$ & 0.91 \\
\hline & $\mathrm{AE}^{1}$ & $\hat{\mathrm{Y}}=\overline{\mathrm{Y}}=4.648$ & - \\
\hline \multirow[t]{3}{*}{ CV-07 } & $\mathrm{TE}^{2}$ & $\hat{\mathrm{Y}}=-0.0006 * \mathrm{P}^{2}+0.0494 * \mathrm{P}+78.068$ & 0.99 \\
\hline & $\mathrm{UE}^{3}$ & $\hat{\mathrm{Y}}=0.1343 * \mathrm{P}+36.389$ & 0.95 \\
\hline & $\mathrm{AE}^{1}$ & $\hat{\mathrm{Y}}=\overline{\mathrm{Y}}=4.980$ & - \\
\hline \multirow[t]{3}{*}{ CV-08 } & $\mathrm{TE}^{2}$ & $\hat{\mathrm{Y}}=\overline{\mathrm{Y}}=77.502$ & - \\
\hline & $\mathrm{UE}^{3}$ & $\hat{\mathrm{Y}}=0.1572 * \mathrm{P}+35.878$ & 0.99 \\
\hline & $\mathrm{AE}^{1}$ & $\hat{\mathrm{Y}}=\overline{\mathrm{Y}}=4.665$ & - \\
\hline \multirow[t]{3}{*}{ CV-09 } & $\mathrm{TE}^{2}$ & $\hat{Y}=\bar{Y}=79.015$ & - \\
\hline & $\mathrm{UE}^{3}$ & $\hat{Y}=\bar{Y}=46.450$ & - \\
\hline & $\mathrm{AE}^{1}$ & $\hat{\mathrm{Y}}=-0.0003 * \mathrm{P}^{2}+0.0585^{*} \mathrm{P}+4.6442$ & 0.90 \\
\hline \multirow[t]{3}{*}{ CV-10 } & $\mathrm{TE}^{2}$ & $\hat{Y}=-0.0706^{*} P^{2}+91.217$ & 0.99 \\
\hline & $\mathrm{UE}^{3}$ & $\hat{\mathrm{Y}}=0.1056^{*} \mathrm{P}+31.437$ & 0.94 \\
\hline & $\mathrm{AE}^{1}$ & $\hat{\mathrm{Y}}=0.0007 * \mathrm{P}^{2}-0.1419 * \mathrm{P}+12.701$ & 0.88 \\
\hline \multirow[t]{3}{*}{ CV-11 } & $\mathrm{TE}^{2}$ & $\hat{\mathrm{Y}}=-0.112 * \mathrm{P}^{2}+91.068$ & 0.87 \\
\hline & $\mathrm{UE}^{3}$ & $\hat{\mathrm{Y}}=0.1940 * \mathrm{P}+21.828$ & 0.93 \\
\hline & $\mathrm{AE}^{1}$ & $\hat{\mathrm{Y}}=0.0004 * \mathrm{P}^{2}-0.0788 * \mathrm{P}+10.623$ & 0.91 \\
\hline \multirow[t]{3}{*}{ CV-12 } & $\mathrm{TE}^{2}$ & $\hat{\mathrm{Y}}=0.001 * \mathrm{P}^{2}-0.2277 * \mathrm{P}+95.861$ & 0.91 \\
\hline & $\mathrm{UE}^{3}$ & $\hat{\mathrm{Y}}=0.1714 * \mathrm{P}+23.309$ & 0.84 \\
\hline & $\mathrm{AE}^{1}$ & $\hat{\mathrm{Y}}=\overline{\mathrm{Y}}=7.583$ & - \\
\hline \multirow[t]{2}{*}{ CV-13 } & $\mathrm{TE}^{2}$ & $\hat{Y}=-0.0602 * P^{2}+89.184$ & 0.99 \\
\hline & $\mathrm{UE}^{3}$ & $\hat{\mathrm{Y}}=0.1486^{*} \mathrm{P}+26.934$ & 0.97 \\
\hline
\end{tabular}

Note: ${ }^{*}$ Significative and ${ }^{\mathrm{n}}$ Non significative by the $\mathrm{t}$ test, at $5 \%$ of probability. ${ }^{1}$ Absorption efficiency $\left(\mathrm{mg} \mathrm{g}^{-1}\right)$;

${ }^{2}$ Translocation efficiency $(\%) ;{ }^{3}$ Utilization efficiency $\left(\mathrm{g}^{2} \mathrm{mg}^{-1}\right)$. 
This fact is justified, as already discussed above, by the genetic and phenotypic differences between conilon coffee clones, as reported by Fonseca et al. (2004) and Ferrão et al. (2008). Different efficiencies for absorption, translocation and utilization of $\mathrm{P}$ in different levels of phosphate fertilizer for genotypes of the same species were also found in potato (Castro, 2009), and corn (Machado et al., 2001), and pepper (Moura et al., 2001) and physic nut (Amaral et al., 2012).

In general, the clones CV-04, CV-10, CV-11 and CV-12 showed higher efficiency of absorption of P (Table 5). These clones were allocated in the groups of lower means of DMR (Table 2) and TP (Table 3), but presented higher P content with a smaller root development when compared to the clones CV-02, CV-07, CV-08 and CV-09 that showed reduced AE, even if those constituted the group of higher means for TDM and TP (Table 2 and 3). According to Fageria (1998), differences in the absorption efficiency of nutrients between genotypes of the same species is basically an adaptation of the root system to the nutritional stress condition.

High translocation efficiency of $\mathrm{P}$ was observed in the clones CV-06 and CV-12, justifiable by the low $\mathrm{P}$ content in roots, unlike the clones CV-02, CV-05, CV-07, CV-08 and CV-09 which had low translocation efficiency of the absorbed phosphorus due to their high values of $\mathrm{P}$ content in the roots (Table 3 ). The translocation efficiency of phosphorus is mainly linked to the genetic characteristic that governs the occurrence of variations in the long-distance transport, the transport speed of the nutrient in the xylem, and the uptake and internal cycling of $\mathrm{P}$ (Furttini Neto, 1994).

In general, for all levels of phosphorus, the clones CV-06, CV-08, CV-09 showed higher UE, contrary to the clones CV-02, CV-04, CV-12 that showed a small capacity of utilization of the absorbed phosphorus (Table 5). The UE was influenced by the ability of accumulate TDM, given that the clones that had highest and lowest UE composed, respectively, the upper and lower mean groups for TDM (Table 2).

Differences in the utilization efficiency of $\mathrm{P}$ among corn cultivars to the same level of phosphorus were found by Machado et al. (2001). These authors state that genotypes that present high utilization efficient of $\mathrm{P}$ are the ones with higher capacity to accumulate dry matter, based on the index proposed by Siddiqi and Glass (1981).

To study the behavior of the $\mathrm{AE}$ as a function of the fertilization with the growing levels of $\mathrm{P}_{2} \mathrm{O}_{5}$, Table 6 shows that the clones CV-01, CV-02, CV-04, CV-10, CV-11 and CV-12 presented fit to linear models. For the clones which exhibit a fit to the regression model, different behaviors are noted in relation to the inflection point of the curves of AE. The clones CV-01, CV-02, CV-04, CV-11 and CV-12 showed efficiency absorption characterized by curves with inflection points in minimal points (Table 6). This fact is mainly due to the lower root development of those clones at the level of $0 \%$ of $\mathrm{P}_{2} \mathrm{O}_{5}$, characterized by lower values of DMR (Table 2). This fact indicates that, despite the lower production of dry matter of root per unit of absorbed $\mathrm{P}$, these clones have a root system very efficient at acquiring $P$ from the soil under conditions of nutritional stress.

Contrary, the curve of absorption efficiency of phosphorus of the clone CV-10 presents characteristic of inflection at a point of maximum (Table 6). Which leads to consider that the root system of this clone has reduced capacity of $\mathrm{P}$ acquisition from the soil under conditions of nutritional stress.

According Tiffney and Niklas (1985), this different behavior between genotypes of the same species is associated with better adaptation to adverse conditions, and also with the distinct ability that they have in change the geometrical configuration of the root system to exploit the resources in a more efficient way.

For AE, the clones CV-03, CV-05, CV-06, CV-07, CV-08, CV-09 and CV-13 didn't present adjustment to any regression model (Table 6), leading to infer that there is no interference of the fertilization with $\mathrm{P}$ in the index of absorption efficiency of these clones, and meaning that the absorption is similar at any level of $\mathrm{P}$.

According to Fageria (1998), genotypes which are similar in nutrients absorption, at different levels of those in the soil, have broad genetic basis controlling the absorption efficiency, showing the adaptation of the roots to the soil nutritional condition. For the current study, this fact becomes interesting, considering the possibility of using the clones CV-03, CV-05, CV-06, CV-07, CV-08, CV-09 and CV-13 in soils considered marginal in $\mathrm{P}$ availability, without significant impairment of the absorption of $\mathrm{P}$.

The TE of the clones CV-03, CV-10, CV-11 and CV-13 were characterized as linear and decreasing (Table 6), an expected fact due to the responses of the $\mathrm{P}$ content in the aerial part and total also present linear characteristic (Table 4). This can be explained by the assertion of Graham (1984), who affirms that the increased of the $\mathrm{P}$ in the soil can suppress the transport rate of $\mathrm{P}$ in the xylem, which reduces the translocation efficiency.

The remaining clones, influenced by the increasing levels of $\mathrm{P}_{2} \mathrm{O}_{5}$ applied in the soil, provide efficiency values for phosphorus translocation adjusted to the quadratic model. The clones CV-01, CV-02, CV-04, CV-06 and 
CV-12 are characterized by curves with inflections at points of minimum and the clones CV-05 and CV-07 showed inflections at points of maximum (Table 6).

Similar results for curves of translocation efficiency of $\mathrm{P}$, with inflections at points of maximum for different pepper genotypes, was found by Moura et al. (2001) and also in soybean by Martinez et al. (1993). These authors suggest that such behavior can be a non limiting mechanism in the metabolic process of $\mathrm{P}$ assimilation, which contributed little to differentiate between strains for response to phosphorus fertilization.

For the clones characterized by curves with inflection at points of minimum, it is assumed that the genetic variability present in these is linked to an adaptation to the environment with conditions of $\mathrm{P}$ stress, due to the more efficient translocation of $\mathrm{P}$ in the absence of phosphorus application in the soil. According Fageria (1998), this relationship has been demonstrated for other vegetables.

The utilization efficiency of phosphorus is linear and crescent in relation to the $\mathrm{P}_{2} \mathrm{O}_{5}$ levels applied in the soil, and the maximum UE of the clones of conilon coffee, except for the CV-09, for which was not possible to adjust a regression model, occurs at the level of $150 \%$ of the recommended level of $\mathrm{P}_{2} \mathrm{O}_{5}$, within the range studied (Table 6).

The fact that the regression model for UE was linear and crescent is basically due the influence of the total dry matter and total $\mathrm{P}$ content in the plant have also showed linear increase as a function of higher levels of $\mathrm{P}_{2} \mathrm{O}_{5}$ in the soil (Table 4), therefore, the clones of conilon coffee showed increase in the biomass production in the same proportion of the accumulation of $\mathrm{P}$ in the vegetal tissues, which increased the UE of phosphorus.

\section{Conclusions}

The clones of conilon coffee have different behavior in relation to the accumulation of dry matter, content of $\mathrm{P}$ in the vegetal tissues, and nutritional efficiency of phosphorus in each level of fertilization with $\mathrm{P}_{2} \mathrm{O}_{5}$.

The dry matter, content of phosphorus and utilization efficiency of phosphorus in clones of conilon coffee increases linearly with the increase of the level of $\mathrm{P}_{2} \mathrm{O}_{5}$ in the soil, under the evaluated conditions.

The absorption efficiency of phosphorus as a function of $\mathrm{P}_{2} \mathrm{O}_{5}$ levels in the soil for the clones of conilon coffee presents fit to a quadratic regression model.

The translocation efficiency of phosphorus in function of $\mathrm{P}_{2} \mathrm{O}_{5}$ levels is differentiated for the clones of conilon coffee, with linear and also quadratic characteristics.

\section{References}

Amaral, J. F. T., Martins, L. D., Laviola, B. G., Christo, L. F., Tomaz, M. A., \& Rodrigues, W. N. (2012). A differential response of physic nut genotypes regarding phosphorus absorption and utilization is evidenced by a comprehensive nutrition efficiency analysis. Journal of Agricultural Science, 4(12), 40-49. http://dx.doi.org/10.5539/jas.v4n12p

Castro, G. Y. (2009). Eficiência de absorção e utilização de fósforo em batata cultivada in vitro. 54f. Dissertação (Mestrado em agronomia), Universidade Federal de Santa Maria, Santa Maria-RS.

Embrapa, Empresa Brasileira de Pesquisa Agropecuária (2006). Centro Nacional de Pesquisa de Solos. Sistema brasileiro de classificação de solos (2 edn) (p. 306). Rio de Janeiro.

Embrapa, Empresa Brasileira de Pesquisa Agropecuária. (1997). Manual de métodos de análises de solo (2 edn) (p. 212). Rio de Janeiro, Ministério da Agricultura e do Abastecimento.

Fageria, N. K. (1998). Otimização da eficiência nutricional na produção das culturas. Revista Brasileira de Engenharia Agrícola e Ambiental, 2, 6-16.

Ferrão, R. G., Cruz, C. D., Ferreira, A., Cecon, P. R., Ferrão, M. A. G., Fonseca, A. F. A., Carneiro, P. C. S., \& Silva, M. F. (2008). Parâmetros genéticos em café Conilon. Pesquisa Agropecuária Brasileira, 43, 61-69. http://dx.doi.org/10.1590/S0100-204X2008000100009

Ferrão, R. G., Ferrão, M. A. G., Fonseca, A. F. A., Lani, J. A., \& Ferrao, L. F. V. (2011). A cafeicultura no estado do Espírito Santo: tecnologias e desafios. In: Tomaz, MA, Amaral, JFT, Jesus Junior, WC, Fonseca, AFA, Ferrão, RG, Ferrão, MAG. (Org.). Tecnologias para a sustentabilidade da cafeicultura. Alegre, ES: CAUFES. 19-50.

Ferreira, D. F. (2008). Sisvar: um programa para análises e ensino de estatística. Revista Symposium, 6, 36-48. 
Fonseca, A. F. A., Ferrão, M. A. G., Ferrão, R. G., Verdin Filho, A. C., Volpi, O. S., \& Zucateli, F. (2004). Conilon Vitória-Incaper 8142: improved Coffea canephora var. kouillou clone cultivar for the state of Espírito Santo. Crop Breeding and Applied Biotechnology, 4, 503-505.

Furtini Neto, A. E. (1994). Eficiência nutricional, cinética de absorção e frações fosfatadas em Eucalyptus spp. 99 f. Tese (Doutorado em Fitotecnia), Universidade Federal de Viçosa, Viçosa-MG.

Gabelman, W. H., \& Gerloff, G. C. (1983). The search for and interpretation of genetic controls that enhance plant growth under deficiency levels of a macronutrient. Plant Soil, 72, 335-350.

Graham, R. D. (1984). Breeding of nutritional characteristics in cereais. In R. B. Tinker, \& C. Lauchlia (Eds.), Advances in plant nutrition (pp. 57-102). New York, Praeger.

Lani, J. A., Prezotti, L. C., \& Bragança, S. M. (2007). Cafeeiro. In L. C. Prezotti, J. A.Gomes, G. G. Dadalto, \& J. A. Oliveira (Eds.), Manual de recomendação de calagem e adubação para o Estado do Espírito Santo (5 aproximação) (pp. 111-118). Vitória: Seea/Incaper/Cedagro.

LI, B., Mckeand, S. E., \& Allen, H. L. (1991). Genetic variation in nitrogen use efficiency of loblolly pine seedlings. Forest Science, 37, 613-626.

Machado, C. T. T., Furlani, A. M. C., \& Machado, A. T. (2001). Índices de eficiência de variedades locais e $\begin{array}{llllll}\text { melhoradas de milho ao fósforo. } & \text { Bragantia, } & 60, & \text { 225-238. }\end{array}$ http://dx.doi.org/10.1590/S0006-87052001000300010

Malavolta, E. (2006). Manual de nutrição mineral de plantas. São Paulo: Agronômica Ceres, 638.

Martinez, H. E. P., Novais, R. F., Sacramento, L. V. S., \& Rodrigues, L. A. (1993). Comportamento de variedades de soja cultivadas sob diferentes níveis de fósforo. II. Translocação do fósforo absorvido e eficiência nutricional. Revista Brasileira de Ciência do Solo, 17, 239-244. http://dx.doi.org/10.1590/S0100-06832003000200017

Moura, W. M., Casali, V. W. D., Cruz, C. D., \& Lima, P. C. (2001). Eficiência nutricional para fósforo em $\begin{array}{lllll}\text { linhagens de } \quad \text { pimentão. } & \text { Horticultura }\end{array}$ http://dx.doi.org/10.1590/S0102-05362001000300002

Novais, F. R., \& Smyth, T. J. (1999). Fósforo em solo e planta em condições tropicais (p. 399). Viçosa: UFV.

Novais, R. F., Smyth, T. J., \& Nunes, F. N. (2007). Fósforo. In R. F. Novais, V. H. Alvarez, N. F. Barros, R. L. F. Fontes, R. B. Cantarutti, \& J. C. L. Neves (Eds.), Fertilidade do solo. Viçosa: Sociedade Brasileira de Ciência de Solo (pp. 471-550).

Oladiran, O., Olajire, F., Robert, C. A., \& Nnenna, I. (2012). Phosphorus response efficiency in cowpea genotypes. Journal of Agricultural Science, 4(1), 81-90. http://dx.doi.org/10.5539/jas.v4n1p81

Reis, T. H. P., Furtini Neto, A. E., Guimarães, P. T. G., Curi, N., Guerra, A. F., \& Marques, J. J. (2011). Dynamics of forms of inorganic phosphorus in soil under coffee plants as a function of successive annual additions of the nutrient. Communications in Soil Science and Plant Analysis, 42(3), 980-991. http://dx.doi.org/10.1590/S0100-06832011000200019

Siddiqi, M. Y., \& Glass, A. D. M. (1981). Utilization index: a modified approach to the estimation and comparison of nutrient utilization efficiency in plants. Journal of Plant Nutrition, 4, $289-302$.

Silva, L., Marchiori, P. E. R., Maciel, C. P., Machado, E. C., \& Ribeiro, R. V. (2010). Fotossíntese, relações hídricas e crescimento de cafeeiros jovens em relação à disponibilidade de fósforo. Pesquisa Agropecuária Brasileira, 45, 965-972.

Swiader, J. M., Chyan, Y., \& Freiji, F. G. (1994). Genotypic differences in nitrate uptake and utilization efficiency in pumpkin hybrids. Journal of Plant Nutrition, 17, 1687-1699. http://dx.doi.org/10.1590/S0100-204X2010000900005

Tiffney, B. H., \& Niklas, K. J. (1985). Clonal growth in land plants: a palaeobotanical prespective. In J. B. C. Jackson, L. W. Buss, \& R. E. Cook (Eds.), Population biology and evolution of clonal organisms (pp. 35-66). New Haven: Yale Universiry Press. 